\title{
Abdominal cystic lymphangioma. A case report
}

\author{
Piotr Szymański, Marcin Zeman, Agnieszka Czarniecka
}

The Oncologic and Reconstructive Surgery Clinic, The Maria Sklodowska-Curie Institute-Oncology Center, Branch in Gliwice, Poland

Introduction. Cystic lymphangiomas are benign tumors that are most prevalent in children in the head and neck region. Abdominal location of these tumors in adults is rare. Radical surgery is the treatment of choice.

Case presentation. We present a diagnostic and therapeutic approach to cystic lymphangioma. A 20-year-old male patient was diagnosed with an extensive multicystic lesion involving the hepatic hilum, hepatoduodenal ligament, aorta and inferior vena cava. Histological examination confirmed the diagnosis of cystic lymphangioma. Non-radical resection was performed as it was impossible to dissect the lesion from large vessels. The patient underwent a consecutive follow-up. Results. An increase in residual lesions was not observed during a 7-year follow-up. No clinical manifestations were reported. Conclusions. Non-radical resection can be considered in the case of cystic lymphangioma if total tumor resection is associated with a high risk of serious intra- and postoperative complications.

NOWOTWORY J Oncol 2019; 69, 1: 36-38

Key words: abdominal cystic lymphangioma, non-radical surgery

\section{Introduction}

Cystic lymphangioma is a tumor arising from lymphatic vessels. It is mostly benign, however, it may be also locally malignant. It is most prevalent in children. Around $65 \%$ of cystic lymphangiomas occur at birth and $90 \%$ occur in patients aged $<2$ years [1]. This tumor is rare in adults. Ninety five percent of lymphangiomas are located in the head and neck [1, 2]. Abdominal lymphangioma is rare (1-5\%) and most cases (80\%) are related to the small bowel mesentery [3]. However, it may occur in any part of the gastrointestinal tract and may be detected in the retroperitoneal space, spleen, liver, gall bladder, adrenal glands, pancreas and the lesser omentum. Studies also reported simultaneous occurrence of lymphangioma in the abdominal cavity and mediastinum [4]. Clinical presentation can be asymptomatic, chronic with non-specific symptoms associated with the mass effect (lumbar pain, vomiting, bowel movement disorder) or acute symptoms related to intestinal obstruction [3], infection of the cystic mass, or bleeding from/ into the tumor [5]. Cases of gastrointestinal bleeding were also reported $[6,7]$. The condition is more prevalent among men (75\%).

\section{Case presentation}

We present a case of a 20-year-old man diagnosed due to lumbar pain lasting for 2 months. Physical examination revealed no abnormalities. Chest X-ray was unremarkable. Abdominal ultrasound revealed a multicystic lesion located intraperitoneally on the right side. Computed tomography and magnetic resonance imaging revealed a large mass $(16.5 \times 8.5 \times 8.5 \mathrm{~cm})$ with the presence of thin septa on the right side of the abdomen, well circumscribed in the lower part and poorly circumscribed from the liver hilum structures, penetrating liver lobes, compressing the duodenum and the head of the pancreas that was in close relation with the aorta and the inferior vena cava (Fig. 1). Ca19-9, CEA, AFP and B-HCG markers were within the normal ranges. The patient was eligible for surgery. Diagnostic laparoscopy showed a cystic tumor located between the right kidney and the liver, extending to the pancreas and duodenum that spread along the hepatoduodenal ligament into the liver hilum. Several cysts filled with slightly turbid fluid were removed. The fluid was sent for cytological, bacteriological and biochemical analysis. The walls of the cysts were sent for intraoperative histological examination which showed 

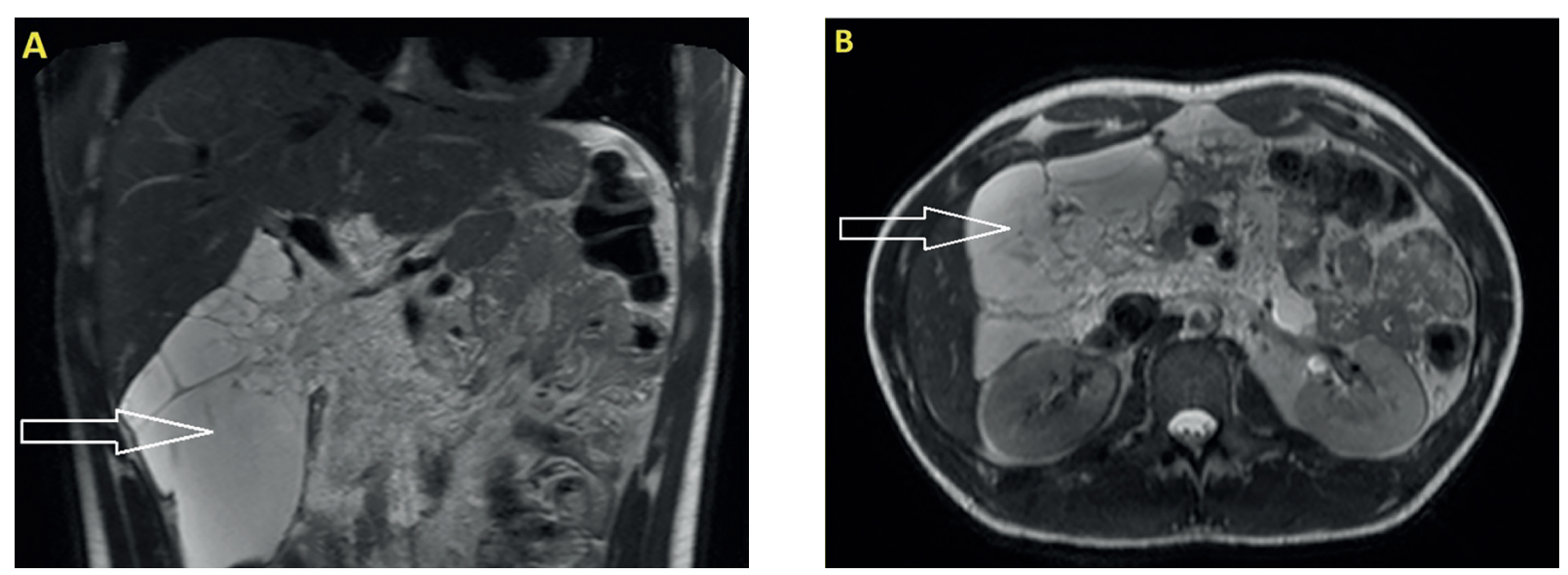

Figure 1. Abdominal magnetic resonance imaging (MRI) before surgery. An extensive multicystic lesion in the right upper abdomen. T2-weighted images; a - coronal section, b - transverse section

fragments of adipose and connective tissues with multiple spaces that might correspond to lymphatic vessels. The result was inconclusive

Cytological examination of the fluid showed the presence of lymphocytes, eosinophils, macrophages containing hemosiderin, endothelial cells and protein masses. Biochemical analysis of the fluid showed the following composition: triglycerides $109 \mathrm{mg} / \mathrm{dl}$, total protein $37 \mathrm{~g} / \mathrm{l}$ and amylase activity $21 \mathrm{U} / \mathrm{l}$. Bacteriological examination of the fluid showed negative culture results. Conversion to laparotomy was performed due to the difficulties in the accurate assessment of the tumor. Most of the tumor was removed. However, due to the fact that it was impossible to dissect the mass from hepatoduodenal ligament structures and large vessels, this part of the tumor was left. Postoperative histological examination revealed multiple cystically dilated vessels (most likely lymphatic) and an irregular distribution of smooth muscle bundles. After the immunohistochemical examination, which confirmed the presence of the CD34 antigen, the final histological diagnosis revealed cystic lymphangioma. The postoperative course was uncomplicated and the patient was discharged in good general condition on day 5 after the procedure. During a 7-year follow-up, several abdominal imaging examinations were performed, which showed a stable residual lesion (Figs. 2, 3).

\section{Discussion}

Differential diagnosis of retroperitoneal cystic tumors includes cystic lymphangioma, mucinous cystadenoma, cystic teratoma, cystic mesothelioma, Mullerian cyst, epidermoid cyst, tailgut cyst, bronchogenic cyst, cystic lesions in solid neoplasms, pseudomyxoma retroperitonei and perianal mucinous carcinoma [8]. Computed tomography and magnetic resonance imaging are the most useful tools in the assessment. A fine-needle aspiration biopsy can be of value [9].

When abdominal lymphangioma is diagnosed, the treatment of choice is total resection by laparotomy or laparoscopic-assisted approach $[1,7]$. However, it is not always possible. The question arises whether in cases requiring extensive organ resection, tumor should always be resected in total. Non-radical resection with no local recurrence during several years of follow-up was reported [10]. In such cases it seems reasonable to perform non-radical resection with a follow-up (as in the case of our patient) or with additional sclerotherapy of residual lesions [11]. Many substances
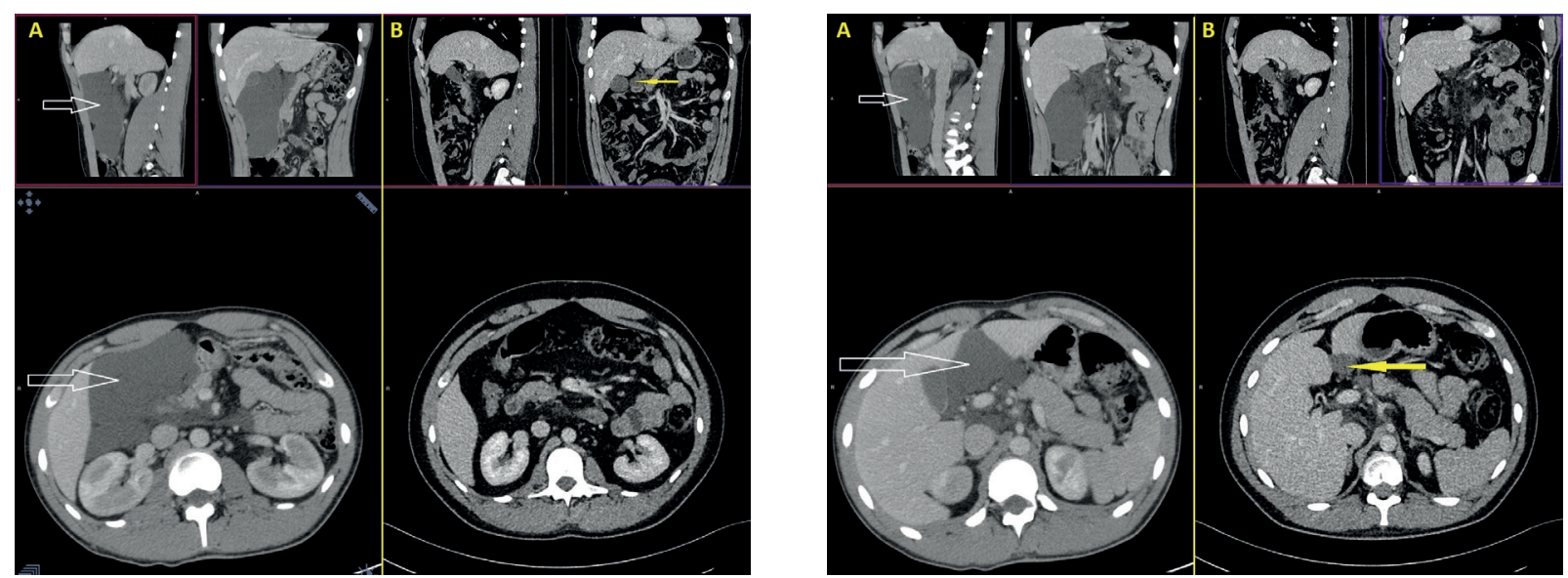

Figures 2, 3. Abdominal computed tomography. Comparison of images before surgical treatment (a) and a follow-up examination - 7 years after surgery (b). The arrow indicates residual lymphangioma 
(e.g. bleomycin, doxycycline, polidocanol, zein solution, OK-432 or fibrin glue) are successfully used in sclerotherapy of lymphangiomas which can be additionally given during surgery or percutaneous drainage. These substances can be also applied as primary treatment alone $[4,12,13]$. Single cases of successful treatment of lymphangiomas using oral drugs, such as propranolol [14] and sirolimus [15], were reported. However, the effectiveness of these drugs has not been fully confirmed yet due to the small number of cases and short follow-ups.

\section{Conclusions}

Our case and the reports of other authors show that total tumor resection which could expose patients to extensive multi organ resection and complications should not be performed in each case. Non-radical resection with a consecutive follow-up is a reasonable approach in such cases.

\section{Acknowledgments}

We wish to thank Arkadiusz Badziński DHSc for the assistance in the translation of the manuscript.

\section{Conflict of interest: none declared}

\section{Piotr Szymański}

The Maria Sklodowska-Curie Institute - Oncology Center Branch in Gliwice

The Oncologic and Reconstructive Surgery Clinic

ul. Wybrzeże AK 15

44-101 Gliwice, Poland

e-mail: szymanskipiotr@mp.pl

Received: 11 Feb 2019

Approved for printing: 8 Mar 2019

\section{References}

1. Kogo H, Matsumoto S, Uchida E. Single-port laparoscopic-assisted resection for a large abdominal cystic lymphangioma: a case report. Surg Case Rep 2018; 4 (1): 92.

2. Serrano-Rodríguez P, Desai CS. Gastrocolic omental cyst in an adult: Case presentation and review of literature. Cir Cir. 2016; 84: 509-512.

3. Khan K, Kleess K, Ganga R et al. Small bowel lymphangioma causing ileo-ileal intussusception in adults. Int J Surg Case Rep; 41: 469-472.

4. Zhou S, Dong S, Du J. Percutaneous therapy of a mediastinal lymphangioma with fibrin glue: case report with clinical success after 4 years. BMC Surg 2018; $18: 4$.

5. Okamoto $\mathrm{D}$, Ishigami $\mathrm{K}$, Yoshimitsu $\mathrm{K}$ et al. Hemorrhagic mesenteric cystic lymphangioma presenting with acute lower abdominal pain: the diagnostic clues on MR imaging. Emerg Radiol 2009; 16: 327-330.

6. Shou-jiang T, Feriyl B. Small Bowel Lymphangioma. Video Journal and Encyclopedia of GI Endoscopy 2014; 1: 663-665.

7. Griffa B, Basilico V, Feltri M et al. Submucosal jejunal lymphangioma: an unusual case with obscure gastrointestinal bleeding in an adult, detected by video-capsule endoscopy and treated by laparoscopy. Minerva Chir 2006; 61: 529-532.

8. Yang DM, Jung DH, Kim H et al. Retroperitoneal cystic masses: $\mathrm{CT}$, clinical and pathologic findings and literature review. RG 2004; 24: 1353-1365.

9. Siderits R, Ouattara O, Abud A et al. Retroperitoneal cystic abdominal lymphangiomatosis diagnosed by fine needle aspiration. Acta Cytol 2009; 53: 191-194.

10. Allen JG, Riall TS, Cameron JL et al. Abdominal lymphangiomas in adults. J Gastrointest Surg 2006; 10: 746-751.

11. García Carretero R, Rodriguez-Maya B, Vazquez-Gomez O. Non-surgical treatment of a relapsed cystic hygroma in an adult. BMJ Case Rep 2017 doi: 10.1136/bcr-2016-218783.

12. Acevedo JL, Shah RK, Brietzke SE. Nonsurgical therapies for lymphangiomas: a systematic review. Otolaryngol Head Neck Surg 2008; 138:418-24.

13. Golinelli G, Toso A, Borello $G$ et al. Percutaneous sclerotherapy with OK-432 of a cervicomediastinal lymphangioma. Ann Thorac Surg 2015; Nov; 100: 1879-1881.

14. Ozeki M, Fukao T, Kondo N. Propranolol for intractable diffuse lymphangiomatosis. N Engl J Med 2011; 364: 1380-1382.

15. Amodeo I, Colnaghi M, Raffaeli $G$ et al. The use of sirolimus in the treatment of giant cystic lymphangioma: four case reports and update of medical therapy. Medicine (Baltimore) 2017; 96: e8871. 